\title{
ESTUDIO COMPARATIVO DE LAS FUNCIONES EJECUTIVAS ENTRE PACIENTES CON ENFERMEDAD DE PARKINSON Y PACIENTES CON ENFERMEDAD DEGENERATIVA CEREBELOSA
}

\author{
Carlos G. Abel', Gustavo Stein', Silvia Pereyra', Gabriela Nano', Tomoko Arakaki', \\ Nélida Garretto', Carlos Mangone², Osvaldo Genovese', Roberto E.P. Sica'
}

RESUMEN - Objetivo: Comparar el rendimiento en tareas de función ejecutiva (FE) en sujetos con enfermedad de Parkinson (EP) leve a moderada (Hoehn y Yahr $\leq 3$ ) no dementes y en sujetos con enfermedad degenerativa cerebelosa pura (EC), a fin de evaluar el eventual rol diferencial de los ganglios basales y el cerebelo en esa función. Método: Se evaluaron 14 pacientes con EP y 14 con EC apareados por sexo, educación, duración de enfermedad y MMSE, mediante tests neuropsicológicos estándar y el test de Wisconsin [WCST]). Se compararon puntajes Z promedio de cada grupo mediante prueba de " $t$ " para muestras independientes. Resultados: El grupo EC rindió significativamente menos en pruebas de atención y FE presentando errores perseverativos y no perseverativos durante el WCST. El grupo EP mostró aumento significativo de errores no perseverativos en relación al estándar esperado, sin alcanzar diferencias significativas con el grupo EC. Conclusión: el grupo EC mostró defectos significativamente mayores en tareas de FE exhibiendo un patrón de disfunción prefrontal.

PALABRAS-CLAVE: cerebelo, ganglios basales, enfermedad de Parkinson, cognición, disfunción ejecutiva, test de Wisconsin.

\section{Comparison study of executive functions in Parkinson's disease and degenerative cerebellar disease's patients}

\begin{abstract}
Objective: To compare executive functions (EF) in non-demented mild to moderate Parkinson's disease (PD) (Hoehn and Yahr $\leq 3$ ) and pure degenerative cerebellar disease (CD) in order to evaluate the relative contribution and differential role of basal ganglia and cerebellum in those functions. Method: 14 patients with PD and 14 patients with CD matched by sex, education, disease's duration and MMSE were selected. A standardized neuropsychological battery and the Wisconsin Card Sorting Test (WCST) were administered. Z scores were compared for both groups through t-test for independent samples were used. Results: The cerebellar group showed significant lower performance in measures of attention and EF, with a significant increase in both perseverative and non perseverative errors during the WCST. On the other hand the PD group showed a selective increase of non perseverative errors, without reaching significant between group difference. Conclusion: The CD group appears to have greater deficits in EF with a pattern of prefrontal dysfunction.
\end{abstract}

KEY WORDS: cerebellum, basal ganglia, Parkinson's disease, cognition, executive dysfunction, Wisconsin test.

La función ejecutiva (FE), término conceptualizado por Luria' ${ }^{1}$ utilizado por Lezak², incluye procesos cognitivos complejos tales como la anticipación, la generación de estrategias, la selección y ejecución del plan de acción apropiado y socialmente aceptable a la meta propuesta, el monitoreo de la conduc- ta manteniendo la estrategia elegida en caso de ser exitosa y flexibilidad mental para variar de estrategia ante contingencias.

Estudios clínicos y experimentales durante las últimas 3 décadas sugieren que en la regulación de las $\mathrm{FE}$, además del rol clásico de la corteza prefrontal ${ }^{3}$,

'División Neurología del Hospital Ramos Mejía, Buenos Aires, Argentina; ²Departamento de Neurología, Hospital Santojanni, Buenos Aires, Argentina.

Received 16 January 2006, received in final form 15 April 2006. Accepted 5 June 2006.

Dr. Carlos Gustavo Abel - División Neurología, Hospital Ramos Mejía - Urquiza 609 - 1221 Buenos Aires - Argentina. E-mail: dakar58@ arnet.com.ar 
participarían estructuras subcorticales tales como los ganglios basales ${ }^{3}$ y el cerebelo ${ }^{4}$, modificando la visión funcional clásica de los ganglios basales y del cerebelo como estructuras vinculadas solo con la coordinación del movimiento.

Dado que en un estudio previo de nuestro gru$\mathrm{po}^{4}$ hemos hallado alteraciones principalmente disejecutivas y visuoespaciales en sujetos con enfermedad cerebelosa pura (EC), nos propusimos comparar el rendimiento en este tipo de tareas en pacientes no dementes con enfermedad de Parkinson (EP), modelo de disfunción de los ganglios basales, y en pacientes con EC a fin de evaluar la contribución relativa de los ganglios basales y el cerebelo en las FE.

\section{MÉTODO}

Pacientes - Se estudiaron durante el año 2002, en la División Neurología del Hospital Ramos Mejía, 28 pacientes, divididos en dos grupos: 14 con el diagnóstico de EP y 14 con diagnóstico de EC, apareados por sexo, educación, valor del MMSE y tiempo de evolución de enfermedad.

Algunos sujetos con enfermedad cerebelosa formaron parte de un estudio previo ${ }^{4}$. La Tabla 1 muestra las características demográficas de cada uno de los grupos de pacientes estudiados.

El estudio no empleó medios invasivos o cruentos, representó una ampliación de la evaluación cognitiva que usualmente se realiza en forma abreviada en pacientes que consultan por ataxia relacionada con degeneración espinocerebelosa y con enfermedad de Parkinson y fue aprobado por los Comité de Docencia e Investigación y de Bioética.

Los criterios de exclusión utilizados para ambos grupos fueron: edad mayor de 70 años, presencia de demencia o de depresión mayor según criterios del DSM-IV 5 o puntaje $\leq 23$ obtenido en el Mini Mental Status Examination (MMSE) ${ }^{6}$, presencia de lesiones isquémicas en los estudios de tomografía computada (TC) o de resonancia magnética de encéfalo (RM), psicosis preexistente, historia de abu-

Tabla 1. Datos demográficos.

\begin{tabular}{lccc}
\hline Datos & $\begin{array}{c}\text { Parkinson } \\
(n=14)\end{array}$ & $\begin{array}{c}\text { Cerebelo } \\
(n=14)\end{array}$ & $\mathrm{p}$ \\
\hline Sexo & $8 \mathrm{M} / 6 \mathrm{~F}$ & $6 \mathrm{M} / 8 \mathrm{~F}$ & $\mathrm{NS}$ \\
Edad (años) & $59,43 \pm 9,94$ & $40,93 \pm 14,95$ & $\mathrm{~S}$ \\
Años de educación & $8,43 \pm 4,91$ & $10,00 \pm 3,92$ & $\mathrm{NS}$ \\
Tiempo de evolución & $7,18 \pm 6,04$ & $10,32 \pm 11,21$ & $\mathrm{NS}$ \\
Hoehn y Yahr & $2,60 \pm 0,56$ & $\mathrm{NA}$ & $\mathrm{NA}$ \\
MMSE & $26,93 \pm 2,50$ & $26,93 \pm 1,49$ & $\mathrm{NS}$ \\
\hline
\end{tabular}

Los puntajes se hallan expresados en medias y desvíos estandar, excepto sexo que se describe como frecuencia. Las medias de edad, educación, tiempo de evolución y MMSE fueron comparadas mediante prueba de t para muestras independientes, excepto sexo que se comparó mediante prueba de Chi cuadrado.M, masculino; F, femenino; NS, no significativo; NA, no aplicable; $S$, Significativo $(p \leq 0.05)$. so de drogas ilícitas o alcohol, traumatismo de cráneo con pérdida de conciencia, antecedentes de infección del sistema nervioso central, enfermedades sistémicas capaces de producir demencia, terapia anticolinérgica, disfunción autonómica, piramidal, extrapiramidal o neuromuscular.

Grupo con EP - Todos los pacientes presentaban parkinsonismo asimétrico, con respuesta adecuada a la levodopa, y cumplían los criterios diagnósticos requeridos por la PDSBB ${ }^{7}$. Los síntomas fueron evaluados por un neurólogo especializado en trastornos del movimiento. Los pacientes que presentaban fluctuaciones fueron estudiados durante la fase "on", coincidiendo con el beneficio clínico motor.

Grupo EC - El diagnóstico de enfermedad cerebelosa degenerativa pura estuvo basado en los hallazgos clínicos y de neuroimágenes (TC y/o RM) con evidencias de atrofia cerebelosa sin otra alteración estructural. Todos los pacientes exhibían leve dismetría en la prueba dedo-nariz. Nueve casos poseían antecedentes familiares de ataxia cerebelosa: 2 casos de atrofia espinocerebelosa tipo 2 (SCA2), 1 caso del tipo 1 (SCA1), 3 casos de origen familiar con un patrón hereditario de tipo autosómico dominante que resultaron negativos para la búsqueda de la alteración genética de las formas SCA 1, 2, 3, 7 y 8; y otros 3, también familiares, que no fueron genéticamente investigados. En los 5 enfermos restantes no se identificaron antecedentes familiares y fueron considerados como padeciendo atrofia cerebelosa primaria adquirida.

Examen neurológico motor - En el grupo EP se utilizó la suma de los puntajes de la subescala motora de la Escala Clínica Unificada de la Enfermedad de Parkinson UPDRS ${ }^{8}$ generando un puntaje de severidad desde 0 , normal, hasta 4, mayor compromiso global y para cada uno de los síntomas mayores. En el grupo EC se uso una escala previamente diseñada para cuantificar la severidad de la ataxia ${ }^{4}$. En ambos grupos se evaluaron las secuencias manuales de Luria (SML) de 3 movimientos (puño, canto y palma) ${ }^{1}$ y las praxias ideomotoras transitivas. A cada una de las escalas motoras diseñadas les fue asignado un puntaje desde 0 , normal, hasta 4, mayor compromiso.

Examen neuropsicológico - La eficiencia cognitiva global se estimó mediante el MMSE${ }^{6}$. La atención y FE se exploraron mediante los subtests de Span de Dígitos (SD) y DígitoSímbolo (DS) de la Escala de Inteligencia de Adultos de Wechsler (Wais III) ${ }^{9}$, los Trail Making Tests (TMTs) partes A (TMTA) y $B(\text { TMTB })^{10,11}$, fluencia verbal ${ }^{12}$, ordenamiento de láminas del WAIS III $(\mathrm{OL})^{9}$ y el test de ordenamiento de cartas de Wisconsin (WSCT) basado en la forma original ${ }^{13}$. Los razonamiento verbal y visual se analizaron mediante el subtest de analogías del Wais $1 \mathrm{II}^{9}$ y las Matrices Progresivas de Raven (MPR) ${ }^{14}$ respectivamente.

Además fueron evaluados el aprendizaje y la memoria verbal mediante el test de Aprendizaje Serial Auditivo Verbal de Rey (RAVLT) ${ }^{15}$, la memoria semántica mediante la prueba de Denominación de Boston (BT) ${ }^{16}$ y la función visual mediante las pruebas de orientación de líneas de 
Benton (LJB ${ }^{17}$, el test de integración visuoespacial de Hooper $(\mathrm{HT})^{18}$ y el subtest de cubos del Wais III ${ }^{9}$.

Análisis estadístico - Los datos obtenidos fueron analizados mediante el programa estadístico SPSS versión 9.0. Los puntajes crudos obtenidos en las diferentes pruebas fueron transformados en puntajes $Z$ y comparados mediante: 1 - prueba de " $\mathrm{t}$ " de una muestra contra 0 para cada grupo; 2 - test de Student de " $\mathrm{t}$ " para muestras independientes entre el grupo EP y EC; 3- pruebas de " $t$ " para muestra apareadas). El análisis de correlación se estableció con el coeficiente de correlación de Pearson ( $r$ ).

\section{RESULTADOS}

El ANCOVA no mostró efecto significativo de la edad en las tareas de $\mathrm{FE}$, razonamiento y habilidad visuoespacial. En cambio, se observó un efecto significativo de grupo en la eficiencia de desempeño en algunas de las tareas relacionadas con estos dominios cognitivos. A pesar de un menor promedio de edad, el grupo EC mostró reducción significativa de la habilidad en dichas áreas cognitivas comparado con el grupo EP.

1. $t$-tests para una muestra contra 0 (Tabla 2, Fig 1) - Ambos grupos presentaron déficits significativos $(p<0.05)$ en relación al estándar esperado para la edad en tareas de FE (TMTs y WSCT). Solo el grupo EC objetivo defectos en el razonamiento (analogías, MPR).
Además ambos grupos mostraron déficits significativos en tareas de memoria (aprendizaje y recuerdo libre verbal del RAVLT) y función visuoespacial (HT).

\section{Comparaciones entre ambos grupos (Tabla 2)}

- El grupo EC mostró eficiencia significativamente menor $(p<0.05)$ para resolver tareas de FE.

Además se observó un rendimiento significativamente menor en tareas de integración visuoespacial (HT) y de razonamiento (Analogías, MPR).

\section{Comparaciones intragrupo}

$E P$ - Comparando el rendimiento en tareas de $\mathrm{FE}$, durante el WCST se observó una dificultad significativamente mayor en la capacidad de mantener una estrategia elegida respecto de la capacidad de variarla debido a un mayor número de errores no perseverativos que de errores perseverativos $(t=2.669, p=$ 0.019).

Paralelamente en tareas de función visuoespacial se observó una eficiencia significativamente menor en integración visuoespacial (HT) comparado con el rendimiento de discriminación visuoespacial (LJB [ $\mathrm{t}=$ 2.440, $\mathrm{p}=0.040]$ ).

$E C$ - La comparación del rendimiento en tareas de FE mostró: 1 - Ausencia de diferencias significati-

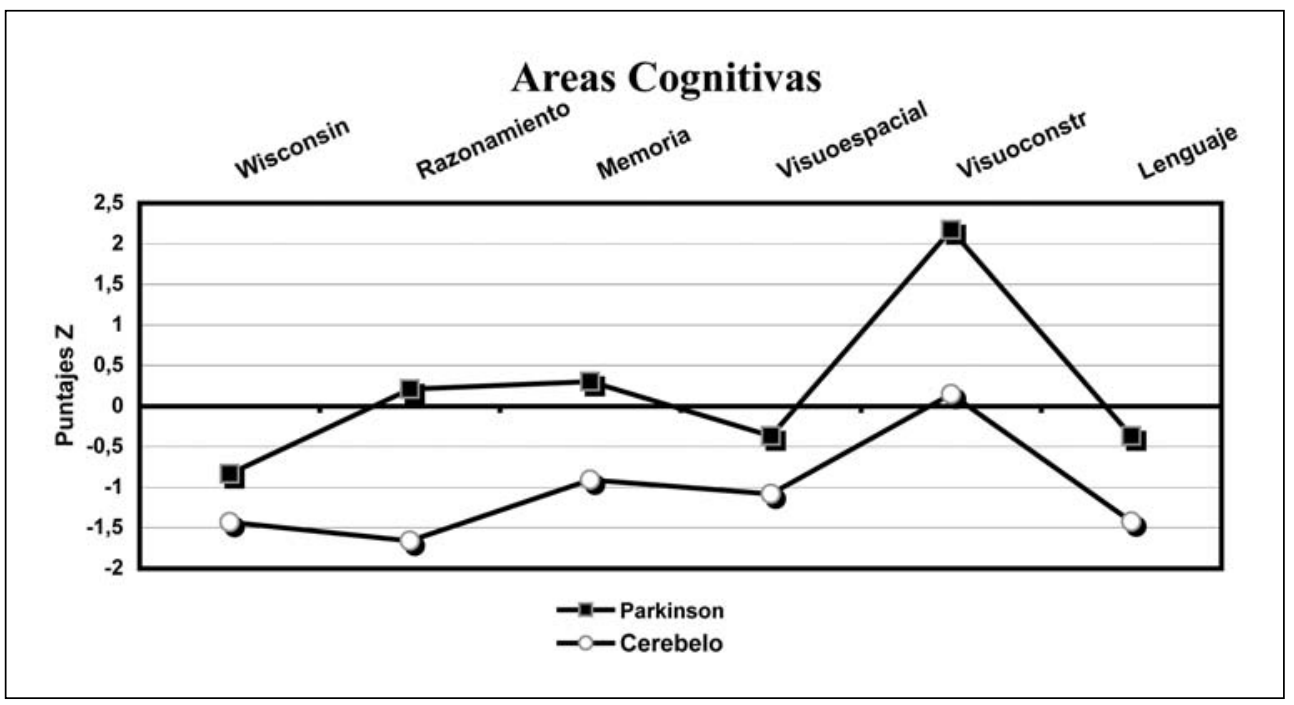

Fig 1. Puntajes $Z$ promedio para los principales dominios cognitivos. FE, Función ejecutiva; WCST, test de Wisconsin; RAVLT, test de aprendizaje auditivo-verbal de Rey; LJB, test de orientación de líneas de Benton; razonamiento representa el rendimiento en razonamientos verbal y visual obtenido por promedio de los puntajes en el subtest de analogias de la escala de WAIS y en Matrices Progresivas de Raven; memoria representa el promedio del rendimiento en aprendizaje, recuerdo libre diferido y reconocimiento del RAVLT; Visuoespacial representa el puntaje obtenido en el LJB; Visuoconstrucción representa el puntaje obtenido en el subtest de Cubos de la escala de WAIS y Lenguaje representa el puntaje obtenido en el test de denominacion de Boston. 
Tabla 2. Rendimientos cognitivo y motor.

\begin{tabular}{|c|c|c|c|c|c|c|c|c|}
\hline \multirow[t]{2}{*}{ Cognicion (Puntajes Z) } & \multicolumn{3}{|c|}{ Parkinson $n=14$} & \multicolumn{3}{|c|}{ Cerebelo $n=14$} & \multicolumn{2}{|c|}{ Comparacion } \\
\hline & \multicolumn{2}{|c|}{ Medias $\pm \mathrm{DE}$} & \multirow[t]{2}{*}{$p$} & \multicolumn{2}{|c|}{ Medias $\pm \mathrm{DE}$} & \multirow[t]{2}{*}{$\mathrm{p}$} & \multirow[t]{2}{*}{$\mathrm{t}$} & \multirow[t]{2}{*}{$\mathrm{p}$} \\
\hline & & & & & & & & \\
\hline Span de Dígitos - WAIS III & $-1,00$ & 3,21 & 0,265 & $-3,50$ & 2,85 & 0,000 & 2,18 & 0,038 \\
\hline \multicolumn{9}{|l|}{ 2- Función ejecutiva } \\
\hline TMTA & $-3,38$ & 2,58 & 0,000 & $-9,72$ & 8,82 & 0,001 & 2,58 & 0,016 \\
\hline ТМтВ & $-2,11$ & 2,76 & 0,029 & $-10,48$ & 10,73 & 0,028 & 2,50 & 0,022 \\
\hline Wisconsin promedio & $-0,83$ & 0,38 & 0,000 & $-1,43$ & 0,60 & 0,000 & 3,15 & 0,004 \\
\hline Errores perseverativos & $-0,11$ & 0,90 & 0,662 & $-1,46$ & 1,21 & 0,000 & 3,38 & 0,002 \\
\hline Errores no perseverativos & $-0,76$ & 0,54 & 0,000 & $-1,08$ & 1,02 & 0,001 & 1,04 & 0,306 \\
\hline Respuestas conceptuales & $-0,52$ & 0,65 & 0,009 & $-1,48$ & 1,08 & 0,000 & 2,85 & 0,008 \\
\hline Categorias completadas & $-1,25$ & 0,39 & 0,000 & $-1,50$ & 0,44 & 0,000 & 1,55 & 0,134 \\
\hline Trials primera categoria & $-1,25$ & 0,41 & 0,000 & $-1,56$ & 0,53 & 0,000 & 1,76 & 0,090 \\
\hline Fallo mantener el set & $-1,33$ & 0,39 & 0,000 & $-1,14$ & 0,36 & 0,000 & $-1,32$ & 0,198 \\
\hline Aprendizaje conceptual & $-1,16$ & 0,30 & 0,000 & $-1,41$ & 0,45 & 0,000 & 1,46 & 0,163 \\
\hline Fluencia fonologica & $-0,61$ & 1,46 & 0,144 & $-0,90$ & 0,95 & 0,003 & 0,63 & 0,535 \\
\hline Fluencia semántica & $-1,09$ & 0,50 & 0,000 & $-1,61$ & 0,64 & 0,000 & 2,42 & 0,023 \\
\hline Dígito-símbolo - WAIS III & 0,50 & 3,18 & 0,566 & $-4,54$ & 1,90 & 0,000 & 4,95 & 0,000 \\
\hline Ordenamiento de láminas - WAIS III & 0,57 & 4,08 & 0,723 & $-3,69$ & 3,33 & 0,001 & 2,53 & 0,020 \\
\hline \multicolumn{9}{|l|}{3 - Razonamiento } \\
\hline Verbal: Analogías - WAIS III & 0,36 & 3,05 & 0,669 & $-2,57$ & 3,78 & 0,024 & 2,26 & 0,032 \\
\hline Visual (MPR) & 0,07 & 1,01 & 0,796 & $-0,75$ & 1,03 & 0,022 & 2,09 & 0,047 \\
\hline \multicolumn{9}{|l|}{ 4- Aprendizaje y Memoria } \\
\hline Aprendizaje verbal - RAVLT & $-1,03$ & 1,26 & 0,009 & $-1,72$ & 1,32 & 0,000 & 1,42 & 0,166 \\
\hline Recuerdo libre verbal - RAVLT & $-0,59$ & 1,02 & 0,049 & $-1,06$ & 1,11 & 0,003 & 1,18 & 0,247 \\
\hline Reconocimiento - RAVLT & 0,71 & 0,49 & 0,000 & 0,03 & 1,14 & 0,918 & 2,03 & 0,053 \\
\hline \multicolumn{9}{|l|}{ 5- Lenguaje } \\
\hline Denominación de Boston & $-0,37$ & 2,12 & 0,531 & $-1,42$ & 2,71 & 0,072 & 1,15 & 0,262 \\
\hline \multicolumn{9}{|l|}{ 6- Habilidad Visual } \\
\hline Visuoespacial (LJB) & $-0,37$ & 1,11 & 0,239 & $-1,08$ & 1,04 & 0,001 & 1,76 & 0,090 \\
\hline Integración visuoespacial (HT) & $-0,51$ & 0,67 & 0,012 & $-1,53$ & 1,33 & 0,001 & $-2,54$ & 0,017 \\
\hline Visuoconstrucción (Cubos) & 2,17 & 3,87 & 0,228 & 0,15 & 2,41 & 0,822 & 1,40 & 0,180 \\
\hline \multicolumn{9}{|l|}{ Motor (Puntajes brutos) } \\
\hline Puntaje motor puro & 2,08 & 0,43 & & 1,58 & 0,66 & & 2,17 & 0,04 \\
\hline Secuencias manuales de Luria & 0,75 & 0,72 & & 1,75 & 1,34 & & $-2,45$ & 0,02 \\
\hline Praxias ideomotoras & 0,22 & 0,47 & & 0,34 & 0,45 & & $-0,69$ & 0,49 \\
\hline
\end{tabular}

Se expresan en medias y desvío estandar para cada prueba y para cada grupo: puntajes Z para las pruebas normatizadas y valores absolutos para las escalas de severidad de compromiso motor. Se compararon las medias obtenidas en cada uno de los tests: 1- mediante prueba de " $t$ " contra 0, 2- entre ambos grupos mediante prueba de " $t$ " para muestras independientes. MMSE, Mini Mental Status Examination; RAVLT, Test de Aprendizaje Serial Verbal de Rey; WAIS III, Escala de Inteligencia para Adultos de Wechsler; MPR, Matrices Progresivas de Raven; LJB, Orientación de líneas de Benton; HT, Test de Integración Visuoespacial de Hooper; DE, desvío estandar; t, puntaje t.

vas entre la capacidad de mantener una estrategia y la capacidad de variarla debido al elevado número tanto de errores no perseverativos como de errores perseverativos durante el WCST ( $t=1.001, p=0.35), 2$ - Eficiencia en FS significativamente menor que en $F F(t=3.526, p=0.004)$.

En tareas de función visuoespacial se observó una eficiencia significativamente menor en integración visuoespacial (HT) comparado con el rendimiento de discriminación visuoespacial (LJB, [t=4.725, $\mathrm{p}=0.000])$.

\section{Correlaciones}

EP (Tabla 3) - El compromiso en la atención y memoria inmediata (SD) correlacionó positivamente con el compromiso en pruebas de FE (TMTA, OL y FF), razonamiento (analogías; MPR) y función visuoespacial (LJB]).

En el análisis de las tareas de FE se observó las siguientes correlaciones significativas y positivas: 1 la- menor cantidad de categorías completadas durante el WCST con una menor eficiencia en el razona- 


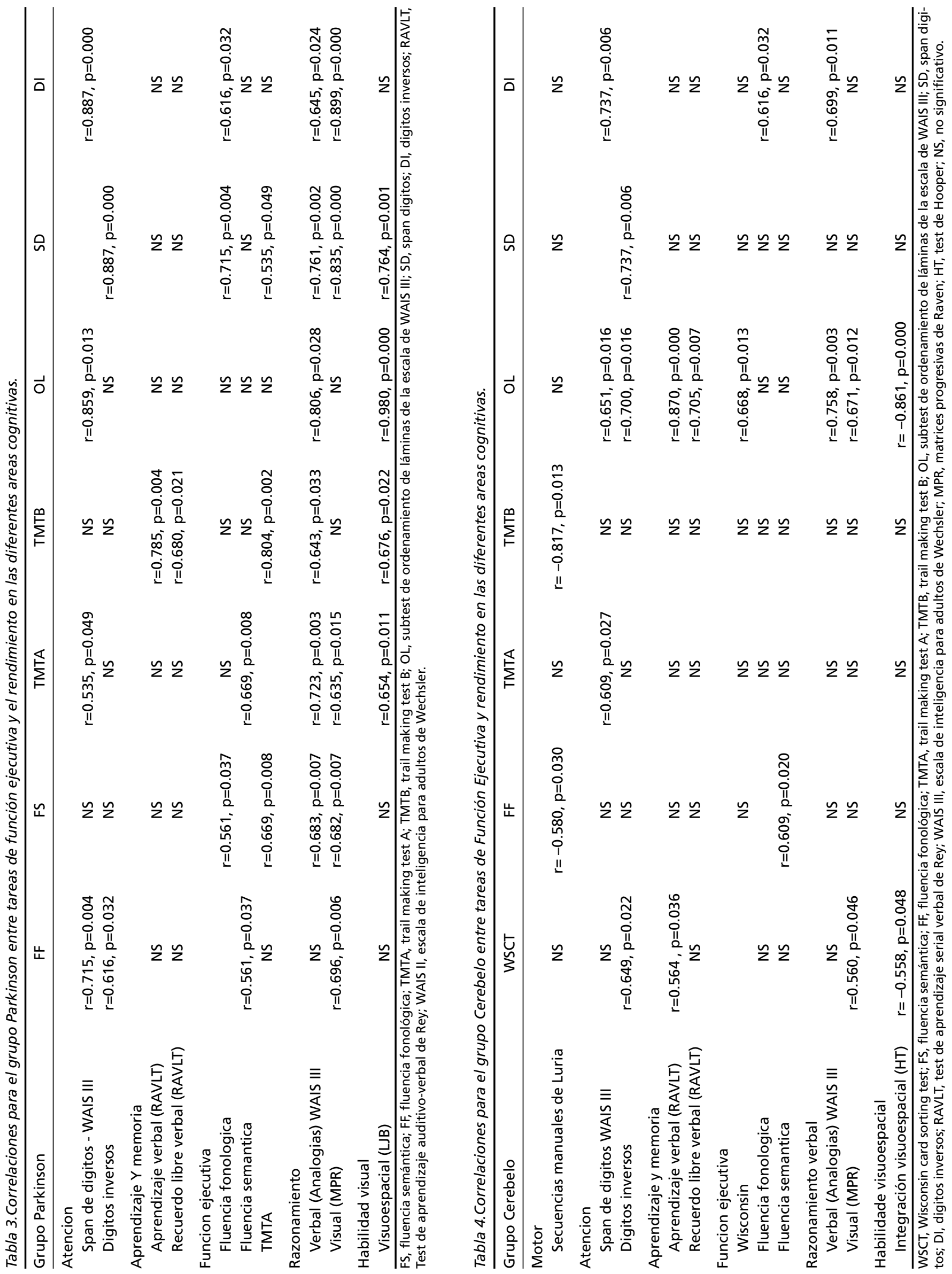


miento (analogías $[r=0.69, p=0.005]$ ) y en la memoria verbal (recuerdo libre diferido: $r=0.67, p=0,008$ ) 2 - las fallas para mantener el criterio con atención y secuenciación (TMTA [ $r=0.644, p=0.013$ ]). 3 - La disminución de la eficiencia en ciertas tareas de atención y FE (SD, TMTA, TMTB, OL) correlacionó con una menor capacidad de discriminación visuoespacial (LJB).

EC (Tabla 4) - Variables cognitivas: La atención correlacionó positivamente con tareas de FE (TMTA, OL). En el análisis de las variables del WSCT se observaron las siguientes correlaciones significativas y positivas: 1 - Mayor numero de errores perseverativos con menor eficiencia en memoria de trabajo verbal (DI $[r=0.62, p=0.040]$ ) y en razonamiento (analogías $[r=$ $0.5, p=0.041] ;$ MPR [ $r=0.61, p=0.024] ; O L$ [ $r=0.57, p=$ $0.039]) 2$ - Menor cantidad de categorías completadas con un menor un rendimiento en $\mathrm{DI}(\mathrm{r}=0.58, \mathrm{p}=$ $0.045)$, en tareas de secuenciación lógico - temporal (OL $[r=0.70, p=0.007]$ y con tareas de integración visuoespacial (HT $[r=0.73, p=0.004])$. Por otro lado el menor rendimiento en ciertas tareas de FE - WCST y especialmente ordenamiento lógico-temporal de la información (OL) - correlacionó positivamente con un menor rendimiento en la habilidad de integración visuoespacial (HT). Variables motoras: Se obser- varon las siguientes correlaciones significativas y negativas: 1 - la severidad de la ataxia con fallo para mantener el set $(r=-0.60, p=0.038) 2$ - los defectos en tareas de secuenciación motora (SML) con un mayor compromiso en ciertas tareas de FE (FF y TMTB) (Tabla 4).

\section{DISCUSIÓN}

Tanto el grupo EP como el grupo EC presentaron un patrón de compromiso cognitivo global leve de tipo fronto-subcortical con déficits significativos en atención, $\mathrm{FE}$, habilidad visuoespacial y recuerdo libre verbal (Tabla 2; Fig 1), hallazgos concordantes con estudios previos ${ }^{3,19}$. Sin embargo, el grupo EC mostró rendimiento significativamente menor en los tests de $\mathrm{FE}$, razonamiento e integración visuoespacial, a pesar de una similar eficiencia cognitiva global entre ambos grupos y de la mayor edad del grupo EP (Tabla 2).

El trastorno significativo del grupo EC en pruebas atencionales (DS, SD y DI) sugeriría la participación del cerebelo en la función atencional, hecho concordante con una investigación previa de nuestro gru$\mathrm{po}^{4}$ y con estudios de resonancia magnética funcional (RMf) que mostraron activación cerebelosa durante tareas de atención selectiva en adultos normales ${ }^{20}$.

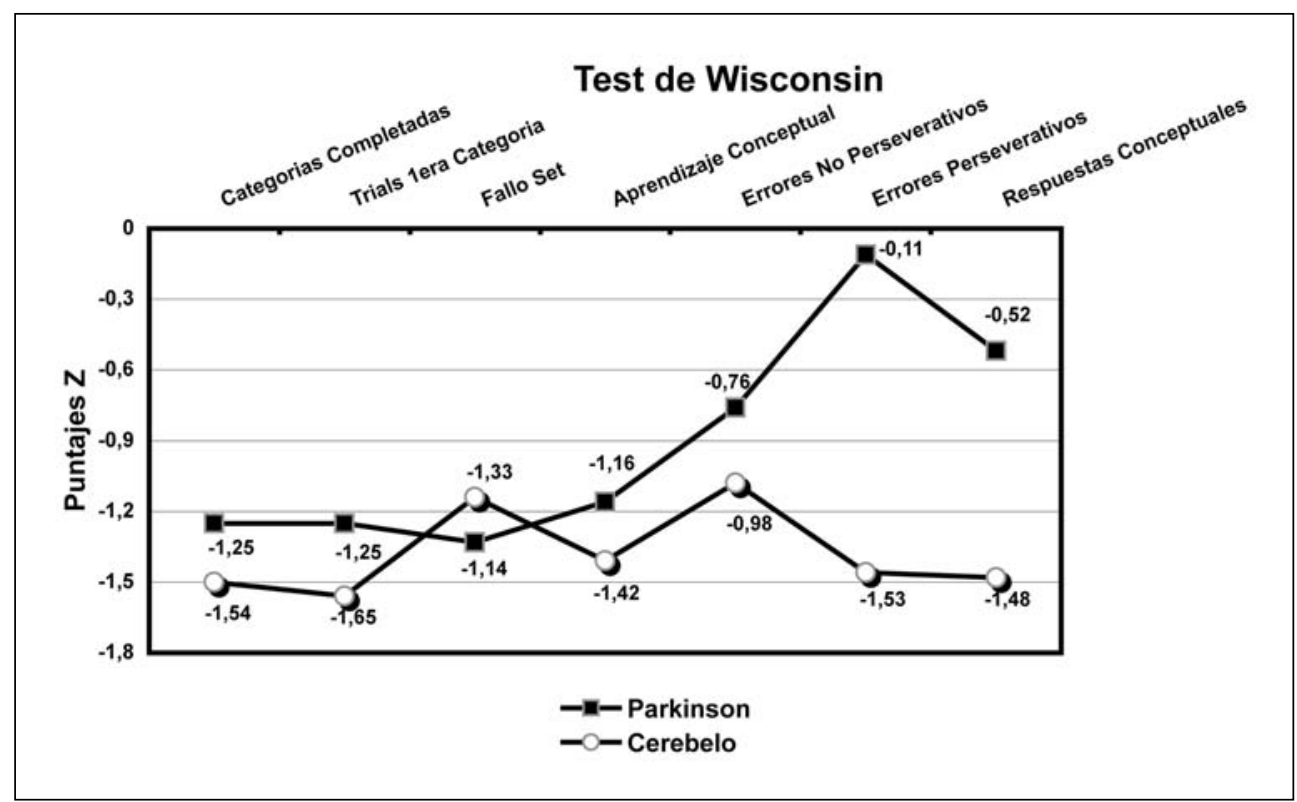

Fig 2. Puntajes $Z$ para cada una de las variables del test de Wisconsin. La prueba de " $t$ " contra 0 demostró una reducción del rendimiento por debajo de lo estimado para el estandar de edad y educación en el puntaje promedio del test de Wisconsin y en cada una de sus variables tanto en el grupo Parkinson como en el grupo Cerebelo excepto en número de errores perseverativos para el grupo Parkinson que rindió apropiadamente en este item. En cambio en la comparación del rendimiento entre ambos grupos el grupo cerebelo mostró un rendimiento significativamente menor puntaje promedio del Wisconsin, en respuestas conceptuales y en errores perseverativos. 
Estos estudios refuerzan la hipótesis que el cerebelo podría optimizar la calidad de la información sensorial al coordinar la dirección de la atención selectiva permitiendo la anticipación, el cambio, la distribución y orientación de la atención según el plan de acción propuesto por la corteza prefrontal ${ }^{20}$.

Ambos grupos mostraron reducción de la fluencia verbal, medida de la FE que explora la flexibilidad cognitiva espontánea al evaluar la habilidad del sujeto para generar estrategias apropiadas que permitan acceder y organizar la producción de la información en unidades de palabras relacionadas fonológica o semánticamente. La reducción de la fluencia verbal podria relacionarse con un mecanismo disejecutivo debido a la falta de correlación entre el rendimiento en estas pruebas y la severidad del compromiso motor en ambos grupos y a la correlación positiva entre la FF y medidas de atención en el grupo EP. El grupo EC presentó además un rendimiento significativamente menor en $\mathrm{FS}$ en relación al grupo EP (Tabla 2) y a su propio desempeño en FF. El compromiso en la FS en el grupo EC concuerda con estudios funcionales, que han demostrado la participación del cerebelo durante tareas de activación semánticas ${ }^{21} \mathrm{y}$ durante tareas de memoria de trabajo verbal, habiéndose descrito la existencia de correlación positiva entre la activación del lóbulo frontal izquierdo y el cerebelo derecho en asociación con el incremento de la demanda en la memoria de trabajo ${ }^{22}$. La disrupción de las conexiones reciprocas cerebelo temporales y prefronto cerebelosas podría afectar el rendimiento en las tareas de FS por disfunción del componente posterior lingüístico dado por la región temporal superior polimodal asociada al procesamiento de la información semántica y por disfunción del componente anterior ejecutivo dado por una red frontosubcortical que incluiría el cerebelo involucrada en la generación de estrategias de búsqueda y recuperación de la información ${ }^{21,22}$.

Ambos grupos mostraron reducción significativa del rendimiento en las pruebas WSCT y de TMTB, que exploran la flexibilidad cognitiva reactiva manifestada por la aptitud de variar una determinada predisposición conductual frente a cambios contingentes del entorno (Tabla 2, Fig 2). Durante el WCST ambos grupos mostraron: 1 - disociación entre la capacidad para verbalizar la respuesta correcta y la capacidad de usar dicha información y ejecutar la respuesta adecuada, 2 - reducción significativa en las medidas de habilidad conceptual estimada por el número de categorías completadas y ensayos para alcanzar la primera categoría y en la capacidad para mantener una estrategia estimada por el número de errores no perseverativos y de fallos para mantener el set.

El bajo rendimiento en el WSCT en nuestro grupo EP coincide con descripciones previas en sujetos con $\mathrm{EP}^{3}$ que señalan una reducción significativa en el número de categorías completadas y un incremento de los errores perseverativos comparados contra controles ${ }^{3}$, con una disociación entre el "saber como hacer" y el "hacer" o ejecutar la respuesta correcta. En cambio nuestro grupo EP mostró un número significativo de errores no perseverativos siendo el número de errores perseverativos el esperado según el estándar de edad y educación. Las diferencias según los diferentes estudios en el número de errores perseverativos en pacientes con EP podrían depender del estadio de enfermedad de los sujetos evaluados $y$, consecuentemente, del grado de compromiso de los circuitos prefronto estriatales. Esta hipótesis fisiopatogénica ha recibido apoyo de un modelo computacional que describe que los sujetos con EP tendrían un elevado número de errores no perseverativos por disfunción estriatal relacionada con la desconexión de los ganglios basales ${ }^{23}$. La mayor degeneración del sistema dopaminérgico nigroestriatal en relación al sistema mesocorticolímbico durante el estadio temprano de la EP produciría mayor disfunción del circuito fronto putaminal que une el área motora suplementaria con el putamen ${ }^{24}$. Dado que este circuito se relaciona con el mantenimiento y refinamiento de la rutina conductual seleccionada, la disfunción del mismo facilitaría errores de tipo no perseverativo.

El grupo EC presentó además una mayor reducción de la eficiencia conceptual medida por un menor número de respuestas conceptuales y de la flexibilidad cognitiva medida por un número significativo de errores perseverativos que estiman la eficiencia en la inhibición de reglas aprendidas y la habilidad para variar de una estrategia a otra frente a contingencias. El perfil perseverativo del grupo EC es similar al descrito en sujetos con daño de la corteza prefrontal dorsolateral ${ }^{3,19}$ o en sujetos con demencia subcortical, tal como la enfermedad de Huntington caracterizada por una disfunción severa del circuito prefronto-caudado ${ }^{25}$. La posibilidad de mayor disfunción de la corteza prefrontal en el grupo EC es sugerida además por las siguientes observaciones: 1 - la correlación del número de errores perseverativos con DI, una medida de la memoria de trabajo y de la fun- 
ción de la corteza prefrontal dorsolateral, encargada del monitoreo de la información en relación con eventos anteriores almacenados en la memoria de trabajo ${ }^{26}, 2$ - la combinación de un elevado número de errores perseverativos y no perseverativos dado que la corteza prefrontal dorsolateral se activa tanto durante la retroalimentación positiva (mantenimiento del criterio) como durante la retroalimentación negativa (señal que implica la necesidad de variar el criterio) del WCST ${ }^{26}$. Además el predominio de errores perseverativos podría señalar una mayor disfunción de la corteza prefrontal medio dorsolateral y ventromedial debido a que se ha descrito la activación selectiva de esta durante la retroalimentación negativa del WCST ${ }^{26}$. Estas observaciones sugerirían que el cerebelo forma parte de una red neural prefronto subcortical que participa en la elaboración y/o cambio de la estrategia que guiará la conducta y en la selección de una conducta preferencial. Esta hipótesis fisiopatogénica es apoyada por estudios funcionales que comprobaron activación tanto de la corteza prefrontal como del cerebelo ${ }^{27}$ durante la alternancia de criterio en la prueba de WSCT. Por lo tanto en la enfermedad cerebelosa degenerativa el compromiso de aquellos circuitos que involucran el núcleo dentado, el tálamo y la corteza prefrontal lateral podría justificar el perfil perseverativo que presentan estos pacientes. En cambio la mayor disfunción del circuito fronto putaminal, en estadios precoces de la EP, explicaría el predominio de errores no perseverativos.

El grupo EC presentó también una dificultad significativamente mayor que el grupo EP en tareas de secuenciación motora (SML)' ${ }^{1}$, grafomotora (TMTs) y cognitiva (OL). La eficiencia de desempeño en las pruebas de secuenciación con alta demanda motora (SML, TMTs) en ambos grupos estaría posiblemente mas relacionada con un defecto cognitivo que con el trastorno motor elemental dado que: 1 - el rendimiento en estas tareas de secuenciación no correlacionó con el puntaje de severidad de compromiso motor (Parkinsonismo y ataxia) (Tabla 2), 2 - En cambio en ambos grupos la reducción de la eficiencia en las tareas de secuenciación con alta demanda motora correlacionó positivamente con la reducción del rendimiento en tareas cognitivas. Mientras en grupo EP el bajo desempeño en los TMTs correlacionó con un menor rendimiento en tareas de aprendizaje y memoria, razonamiento y capacidad visuoespacial (Tabla 3), en el grupo EC el menor rendimiento en SML correlacionó con una menor eficiencia en tareas de FE (FF y TMTB). Por otro lado, en el grupo
EC, la reducción de la eficiencia en las tareas de secuenciación se mantuvo también en una prueba de secuenciación cognitiva (OL) consistente en el ordenamiento lógico temporal de la información (Tabla 2), la cual a su vez, correlacionó positivamente con tareas de FE y razonamiento (WSCT, Analogías, MPR, $\mathrm{HT}$ ) (Tabla 4). A diferencia del grupo EP, en el grupo EC la reducción del rendimiento durante los TMTs, no correlacionó con una reducción de eficiencia en las demás áreas cognitivas (aprendizaje y memoria, razonamiento y habilidad visuoespacial). Esto podría señalar diferentes mecanismos de falla en cada uno de los 2 grupos: 1 - En el grupo EP, la reducción del rendimiento cognitivo global podría vincularse mas estrechamente con un mecanismo de falla disejecutiva relacionado con la disfunción de circuitos prefronto-estriatales, 2 - En cambio, el grupo EC la reducción del rendimiento cognitivo global, especialmente en razonamiento y habilidad visuoespacial, parece ser independiente de la magnitud del compromiso disejecutivo, aun cuando este sea significativamente mayor que el del grupo EP. Esto indicaría que en el grupo EC existiría una disrupción de diferentes conexiones reciprocas cerebro-cerebelosas comprometiendo circuitos prefronto y temporoparieto cerebelosos. Por otro lado, la mayor dificultad del grupo EC en el ordenamiento secuencial de una serie de eventos o acciones en una unidad coherente de conducta reforzaría la hipótesis de la participación del cerebelo en la representación temporal de eventos motores y no motores ${ }^{28}$, permitiendo el aprendizaje y refinamiento de rutinas conductuales a través de la anticipación, programación y adaptación de las respuestas en tiempo real. Los errores en la selección de la secuencia apropiada de pasos para resolver un problema podría explicar las fallas en la generación de estrategias, planificación, organización temporal de la conducta y el aprendizaje procedural en pacientes con trastornos de los ganglios basales ${ }^{29}$ o del cerebelo ${ }^{19,30}$. Otro mecanismo alternativo para explicar la gran dificultad del grupo EC en la ejecución del TMTB, seria la existencia de un severo compromiso en la capacidad para variar de un programa conductual a otro y estaría de acuerdo con el perfil perseverativo previamente descrito durante el WCST.

Solo el grupo EC presentó reducción significativa del rendimiento en las tareas de razonamiento (Analogías, MPR) (Tabla 2; Figura 1), las que correlacionaron positivamente con medidas de FE (OL, errores perseverativos del WCST) (Tabla 4) sugiriendo la participación de un mecanismo disejecutivo y de inflexibilidad cognitiva como una de las causas posibles 


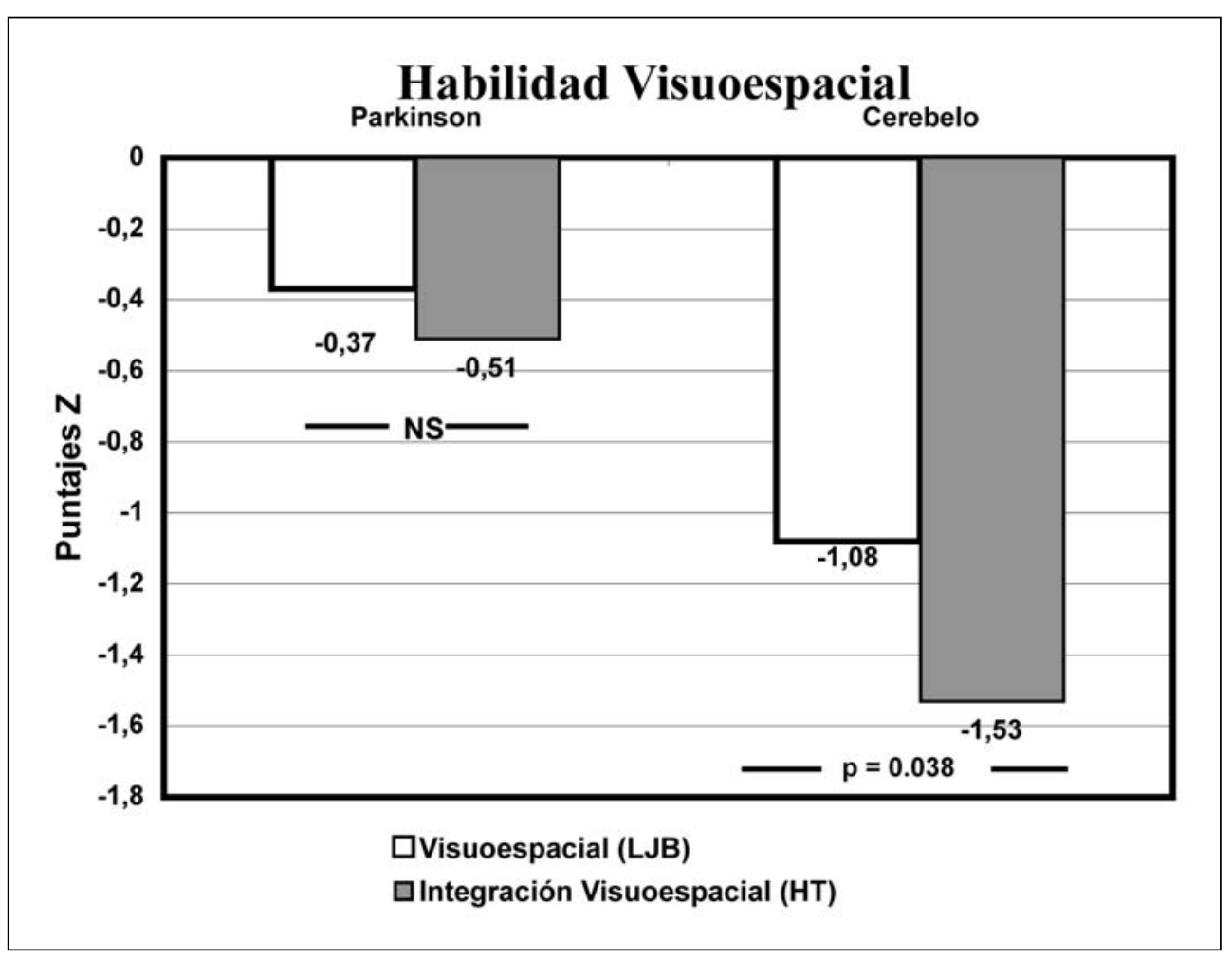

Fig 3. Habilidades visuoespaciales. El grupo Cerebelo mostró un rendimiento significativamente menor en el test de integración visuoespacial de Hooper en relación al grupo Parkinson $(p=0.038)$ no encontrandose diferencias significativas entre ambos grupos en el test de orientacion de lineas de Benton, predominantemente perceptivo. LJB, test de orientacion de lineas de Benton; HT, test de integracion visuoespacial de Hooper.

que llevan a la reducción de la capacidad de abstracción en estos enfermos. Si bien el grupo EP no presentó reducción del rendimiento en las tareas de razonamiento evaluadas, la reducción de la eficiencia conceptual durante el WCST estimada por el número de categorías alcanzadas, sugeriría un compromiso leve del razonamiento lógico abstracto en el grupo EP, al menos en etapas tempranas.

Ambos grupos presentaron reducción significativa en razonamiento visual medido por tareas que miden la habilidad de integración visuoespacial (HT) (Tabla 2; Figura 3). Dado que el HT plantea una mayor demanda ejecutiva al requerir de una apropiada generación de estrategias de búsqueda y reorganización de la información mediante la rotación mental de imágenes de partes de objetos o animales fragmentados $^{18}$ es posible que el bajo rendimiento en esta prueba se relacione con el grado de disfunción ejecutiva presentaban ambos grupos. En el grupo EC el rendimiento en el HT correlacionó positivamente con el rendimiento en tareas de $\mathrm{FE}$ y razonamiento (OL, analogías) y de acceso lexical (BT) (Tabla 4), subcomponentes funcionales requeridos para la adecua- da ejecución del HT. La fisiopatogenia de estas fallas visuoespaciales en el grupo EC podría vincularse con la disrupción de las conexiones prefronto cerebelosas y cerebelo-parietales, que generaría menor eficiencia del procesamiento de la información visuoespacial por interrupción de los circuitos que unen la corteza parietal posterior, involucrada en la función visuoespacial, con la corteza prefrontal dorsolateral, involucrada en la $\mathrm{FE}^{19}$.

Algunos factores potencialmente limitantes de nuestro estudio deben tenerse en cuenta. Aunque el efecto de la levodopa en la función cognitiva de pacientes con EP es aun tema de controversia ${ }^{31}$, la evaluación de nuestros pacientes parkinsonianos en la fase "on", crea un sesgo de potencial beneficio cognitivo medicamentoso relacionado con la mayor disponibilidad de dopamina a nivel del núcleo caudado y del estriado ventral, relacionados con funciones cognitivas y conductuales. Otro aspecto es que si bien todos los pacientes de nuestro grupo EC tenían en común la presencia de enfermedad de causa degenerativa restringida al cerebelo, existe heterogeneidad respecto de las causas genéticas y biológicas mo- 
leculares implicadas en cada uno de estos pacientes. De todos modos se busco comparar, teniendo en cuenta estas limitaciones, dos modelos degenerativos subcorticales con disfunción restringida a dos estructuras subcorticales en particular: los ganglios basales en el caso del grupo EP y el cerebelo en el caso de la enfermedad cerebelosa degenerativa aislada.

En base a nuestros hallazgos y a estudios anteriores ya mencionados, es posible suponer que estructuras subcorticales, como el cerebelo o los ganglios basales, forman parte de una red fronto-subcortical que contribuye, junto a la corteza prefrontal, en la regulación de las FE permitiendo una creciente eficiencia, rapidez y automatización de una tarea, guiando la acción de acuerdo a las reglas propuestas por la corteza prefrontal responsable de la activación de los planes y acciones y de la estructuración lógicotemporal de una secuencia conductual ${ }^{4}$. Estas observaciones refuerzan además la hipótesis de la participación del cerebelo en la planificación cognitiva en forma análoga a como organiza las destrezas motoras que requieren una serie de movimientos individuales integrados para constituir una secuencia unitaria. La disfunción ejecutiva severa ${ }^{19}$ producida por la disfunción cerebelosa puede constituir una causa relevante de alteración funcional que se sumaría al defecto motor elemental y que debería ser considerada al planear la reeducación de estos pacientes.

\section{REFERENCIAS}

1. Luria AR. Higher cortical functions in man. Luria AR (ed). English translation by Haigh B. of $1^{\text {st }}$ Russian edition (Moscow University Press, Moscow, 1962). New York: Basic Books and Plenum Press, 1966.

2. Lezak M. The problems of assessing executive functions. Int J Psychol 1982;17:281-297.

3. Taylor A, Saint-Cyr J, Lang A. Frontal lobe dysfunction in Parkinson's disease. Brain 1986;109:845-883.

4. Abel C, Stein G, Pereyra S, et al. Evaluación cognitiva en 12 pacientes con enfermedad cerebelosa degenerativa pura. Rev Neurol 2005;40;8: 465-472.

5. American Psychiatric Association. Diagnostic and statistical manual of disorders (DSM-IV). Washington, D.C.: American Psychiatric Association, 1994.

6. Folstein M, Folstein S, McHugh P. Mini-mental state: a practical method for grading the cognitive status of patients for the clinician. J Psychiatr Res 1975;12:189-198.

7. Hughes A, Daniel S, Kilford L, Lees A. Accuracy of clinic diagnosis of idiopathic Parkinson's disease: a clinico-pathological study of 100 cases. J Neurol Neurosurg Psychiatry 1992;55:181-184.

8. Fahn S, Elton R, Members of the UPDRS Development Committee. Unified Parkinson's disease rating scale. In Fahn S, Marsden C, Calne
D, Goldstein M (eds). Recent developments in Parkinson's disease, Vol. 2. Florham Park: Macmillan Health Care Information, 1987:153-164.

9. Wechsler D. The Wechsler Adult Intelligence Scale. New York: The Psychological Corporation, 1955.

10. Tombaugh T, Rees L, McIntyre N. Normative data for the Trail making test. In Spreen O, Strauss E (eds). A compendium of neuropsychological test. Administration, norms, and commentary. New York: Oxford University Press, 1998:539-542.

11. Yeudall L, Reddon J, Gill D, Stefanyk W. Normative data for the Halstead-Reitan neuropsychological tests stratified by age and sex. J Clin Psychol 1987;43:346-367.

12. Tombaugh T, Kozak J, Rees L. Normative data for the controlled oral word association test. In Spreen O, Strauss E (eds). A compendium of neuropsychological test. Administration, norms, and commentary. New York: Oxford University Press, 1988:453-457.

13. Heaton R. A manual for the Wisconsin Card Sorting Test Manual. Odessa: Psychological Assessment Resources, 1981.

14. Raven J. Standard progressive matrices. Oxford: Oxford Psychologists Press, 1976.

15. Geffen G, Moar K, O’Hanlon A, Clark C, Geffen L. Performance measures of 16- to 86-year-old males and females on the auditory verbal learning test. Clin Neuropsychol 1990;4:45-63.

16. Allegri R, Mangone C, Rymberg S, Fernandez A, Taragano F. Spanish version of the Boston naming test in Buenos Aires. Clin Neuropsychol 1997;11:416-420.

17. Benton A, Hamsher K, Varney N, Spreen O. Contributions to neuropsychological assesment: a clinical manual. New York: Oxford University Press, 1983:44-54.

18. Hooper H. Hooper visual organization test (VOT) Manual. Los Angeles: Western Psychological Services, 1983.

19. Schmahmann J, Sherman J. The cerebellar cognitive affective syndrome. Brain 1998;121:561-579.

20. Akshoomoff N, Courchesne E, Townsend J. Attention coordination and anticipatory control. Schmahmann J (ed). The cerebellum and cognition. San Diego: Academic Press, 1997:575-598.

21. Petersen S, Fox P, Posner M, Mintun M, Raichile M. Positron emission tomographic studies of the processing of single words. J Cogn Neurosci 1989;1:153-170.

22. Fiez J, Raife E, Balota D, Schwarz J, Raichle M, Petersen S. A positron emission tomography study of the short-term maintenance of verbal information. J Neurosci 1996;16:808-822.

23. Amos A. Computational model of information processing in the frontal cortex and basal ganglia. J Cogn Neurosci 2000;12:505-519.

24. Javoy-Agid F, Agid Y. Is the mesocortical dopaminergic system involved in Parkinson disease? Neurology 1980;40:1326-1340.

25. Albert M, Feldman R, Willis A. The subcortical dementia of progressive supranuclear palsy. J Neurol Neurosurg Psychiatry 1974;37:121-140.

26. Monchi O, Petrides M, Petre V, Worsley K, Dagher A. Wisconsin card sorting revisited: distinct neural circuits participating in different stages of the task identified by event-related functional magnetic resonance imaging. J Neurosci 2001;2:7733-7741.

27. Berman K, Ostrem J, Randolph C, et al. Physiological activation of a cortical network during performance of the Wisconsin card sorting test: a positron emission tomography study. Neuropsychologia 1995;33:10271047.

28. Ivry R, Keele S. Timing functions of the cerebellum. J Cogn Neurosci 1989;1:136-152.

29. Saint-Cyr J, Taylor A, Lang A. Procedural learning and neostriatal dysfunction in man. Brain 1988;111:941-959.

30. Grafman J, Litvan I, Massaquoi S, Stewart M, Sirigu A, Hallett M. Cognitive planning deficit in patients with cerebellar atrophy. Neurology 1992;42:1493-1496.

31. Frank M. Dynamic dopamine modulation in the basal ganglia: a neurocomputational account of cognitive deficits in medicated and nonmedicated parkinsonism. J Cogn Neurosci 2005;17:51-72. 